\title{
EVOLVING A SIMULATED ROBOTIC ARM ABLE TO GRASP OBJECTS
}

\author{
GIANLUCA MASSERA ${ }^{\mathrm{A}}$ STEFANO NOLFI ${ }^{\mathrm{A}}$ ANGELO CANGELOSI ${ }^{\mathrm{B}}$ \\ ${ }^{A}$ Institute of Cognitive Science and Technologies, National Research Council(CNR, \\ Italy) \\ ${ }^{B}$ Adaptive Behaviour \& Cognition Reserch Group (University of Plymouth, UK) \\ gmassera@istc.cnr.it,nolfi@istc.cnr.it, acangelosi@plymouth.ac.uk \\ In this paper we will present the result of a set of experiments in which an robotic arm \\ with a two-fingered hand is evolved for the ability to grasp objects. Obtained results are \\ encouraging and demonstrate that evolutionary robotics techniques might scale up to \\ situations involving robots with several degrees of freedom and problems that require an \\ ability to produce sequential behaviours.
}

\section{Introduction}

In this paper we will present the result of a set of experiments in which a robotic arm with a two-fingered hand is evolved for the ability to grasp objects (for a previous related model, see Bianco and Nolfi, 2004; for other models in which controllers for robotic arms have been developed through evolutionary techniques, see Moriarty and Mikkulainen, 1996; Skopelitis, 2002; Buehrmann and Di Paolo, 2004). Preliminary results demonstrate that the problem of grasping objects in varying environmental conditions can be solved by producing rather simple forms of behaviour. These forms of behaviour exploit properties emerging from the interaction between the body of the robot, its control system and the environment. Evolved neural controllers, however, are not able to master cases in which the position of the objects to be grasped vary significantly. In the following sections we briefly describe: the model and the experimental setup, the obtained results, and the future research directions.

\section{Model and Experimental Setup}

\subsection{The Robotic Arm}

The simulated robot consists of various objects articulated by joints as illustrated in Figure 1. The arm consists of three segments (the arm, the forearm, and the wrist, respectively) that are attached to the previous segments (the shoulder, the arm, and the forearm, respectively) through three joints. Each joint has two degrees of freedom (DOF). The hand consists of two fingers (a 
thumb and an index finger) attached to the wrist with two corresponding joints with one DOF. The index finger also includes an additional segment attached to the previous segment with a joint with one DOF. Therefore the whole arm consists of six motorized joints and six segments with 9 DOF.

To model the physical dynamics as accurately as possible, we used a library for simulating rigid body dynamics called ODE (Open Dynamics Engine www.ode.org).

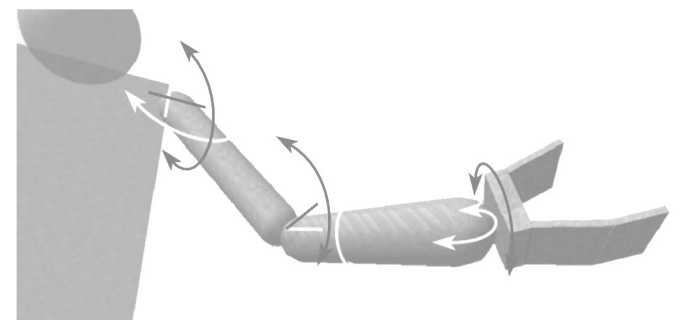

Figure 1. The robotic arm.

\subsection{The Neural Controller}

The neural controller consists of a recurrent neural network with 33 sensory neurons, 2 internal neurons, and 9 motor neurons. Sensory neurons are connected to the internal and motor neurons. Internal neurons are connected to themselves and to the motor neurons. The sensory neurons encode: the current angular position of the joints controlling the 9 DOF ( 9 neurons), the state of 16 contact sensors placed on the fingers and on the forearm (16 neurons), and the current relative position of the target object with respect to the hand ( 8 neurons). The 9 motor neurons control the actuators of the 9 corresponding joints. The output of the neurons is normalized within the range of movement of the corresponding joint and is used to set the desired position of the corresponding joint. More precisely, motors are activated so as to reach a speed proportional to the difference between the current and the desired position of the joint.

\subsection{Genetic Algorithm}

The neural controllers have been evolved (Nolfi and Floreano, 2000) for the ability to grasp objects placed on a planar surface.

The genotype of evolving individuals encodes the connections' weights and the biases of the neural controller. Each parameter is encoded with 8 bits. Weights and biases are normalized between -5.0 and 5.0. Population size is 
100. The 20 best individuals of each generation were allowed to reproduce by generating 5 copies of their genotype which were mutated by flipping $1 \%$ of randomly selected bits.

Each individual of the population was tested for 10 trials, with each trial consisting of 400 steps (each step lasts $15 \mathrm{~ms}$ ). At the beginning of each trial the arm and the hand is set in a similar but randomly varied position (i.e. the initial angles of the joints varies within a $2 \%$ range). After 260 steps the planer surface is removed to allow un-grasped objects to fall down. Individual are selected on the basis of an ability to grasp the objects. To facilitate the emergence of this ability, however, individuals are also rewarded for the ability to touch the objects with their fingers. More precisely, the fitness is calculated according to this formula: $\mathrm{NC}+\mathrm{NG}^{*} 1000$, where $\mathrm{NC}$ is the numbers of contacts between the fingers and the objects occurring during the first 260 time steps, and NG is the number of time steps in which the object is inside the robot's hand during the successive time steps in which the planar surface has been removed.

\section{Results}

We ran four sets of experiments and for each experimental set we ran 10 different replications starting from different randomly generated population of genotypes.

In the first set of experiments evolving individuals were asked to grasp spherical objects located on the right side with respect to the initial position of the arm. By analysing the obtained behaviour we observed that evolved individuals are able to successfully grasp the objects in most of the replications. More specifically, in the case of the best replication, evolved individuals show an ability to successfully grasp objects in $71 \%$ of the cases.

One complex aspect of this task is that, due to the low friction with the table, spheres can easily roll away as a result of contact between the arm and the object thus preventing the possibility to successfully grasp them. Evolved individuals master this complex situation by carefully calibrating their movements and by appropriately orienting their hand while moving toward the object. Indeed, in the best evolved individuals, the grasping behaviour is triggered by internal neurons that detect when the hand is correctly oriented with respect to the object (Fig. 2). 


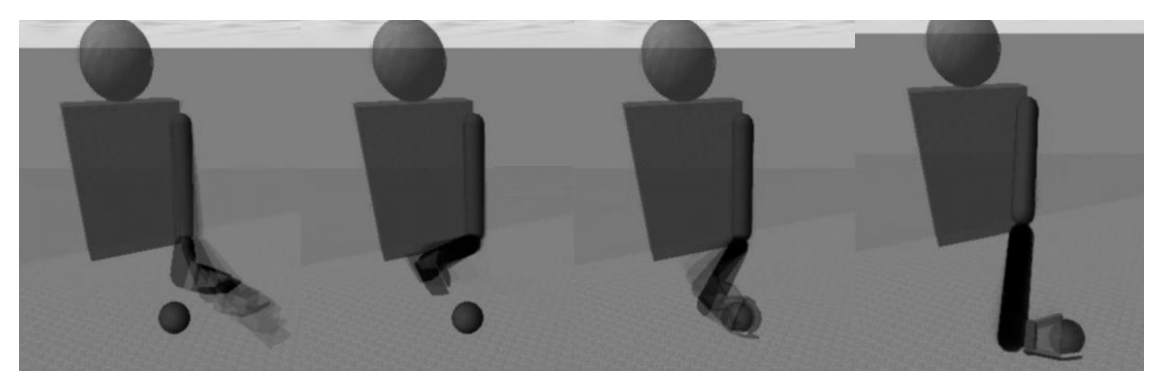

Figure 2. Four snapshots of a typical evolved behaviour. The robot approach the object by orienting its hand appropriately and then successfully grasp the sphere.

In the second set of experiments evolving individuals were asked to grasp cubic objects located on the right side with respect to the initial position of the arm. By analysing the obtained behaviour we observed that evolved individuals are able to successfully grasp the objects in most of the replications. More specifically, in the case of the best replication, evolved individuals show an ability to successfully grasp objects in the $87 \%$ of the cases.

Cubic objects do not tend to roll away as easily as spheres. As a consequence, evolved robots approach the objects through fast movement and hit them so that they start to move. The dynamics of the interaction between the moving object and the moving hand is exploited in order to modify the relative orientation of the object until an effective grasping is obtained.

In the last two sets of experiments evolving individuals were asked to grasp objects located both on the left and the right side with respect to the initial position of the arm. These two experimental sets differ with respect to the type of objects (spheres or cubes) to be grasped.

Obtained performance were much worse in both sets of experiments and no evolved individual displayed an ability to grasp objects located in both sides. Indeed, evolving individuals tended to specialize on only one of the two sides.

\section{Discussion and Future Research Directions}

Obtained results are encouraging and demonstrate that evolutionary robotics techniques might scale up to situations involving robots with several degrees of freedom and problems that require an ability to produce sequential behaviors.

However, we also showed that evolved individuals are not capable of successfully grasping objects placed in significantly different positions. This failure might be explained by considering that grasping objects located in significantly different positions requires the ability to display qualitatively 
different sequential behaviours.

To tackle this problem in future research we plan to provide evolving neural controllers with additional internal units that might be used to categorize different environmental situations and modulate the behaviour of the basic neural controller accordingly so that qualitatively different sequential behaviour can be produced in different environmental circumstances.

In particular, we are interested in robots able to develop dynamical internal categories, that is internal states that tend to vary while the robot is interacting with the environment by producing different dynamical paths in different environmental situations. Dynamical categories of this form might not only allow robots to deal with situations requiring the ability to produce qualitatively different sequential behaviours but would also constitute an abstract high-level description (dynamically coupled with the sensory-motor flow) of the sequence of micro-movements required.

\section{Acknowledgments}

This research has been supported by the grant FIRB:RBNE01SZB4 of the Italian Ministry of Education, University and Research (MIUR) and by the ECAgents project funded by the Future and Emerging Technologies program (IST-FET) of the European Commission under the EU RD contract IST-1940.

\section{References}

Bianco R., Nolfi S. (2004). Evolving the neural controller for a robotic arm able to grasp objects on the basis of tactile sensors. Adaptive Behavior, 12(1):37-45.

Buehrmann T. and Di Paolo E.A., (2004). Closing the loop: Evolving a model-free visually-guided robot arm. Proceedings of the Ninth International Conference on the Simulation and Synthesis of Living Systems, ALIFE'9 Boston, September 12th-15th, 2004. MIT Press

Moriarty D.E., Mikkulainen R. (1996), Evolving obstacle avoidance behavior in a robot arm, In Maes P., Mataric M., Meyer J.-A., Pollack J., and Wilson S.W (eds.), Proceedings of the Fourth International Conference on Simulation of Adaptive Behaviors. Cambridge MA: MIT Press.

Nolfi S., Floreano D. (2000). Evolutionary Robotics: The Biology, Intelligence, and Technology of Self-Organizing Machines. Cambridge, MA: MIT Press/Bradford Books

Skopelitis C. (2002), Control System for a Robotic Arm, Master Thesis, School of Cognitive and Computing Sciences (COGS), University of Sussex, U.K. 